\title{
Developmental Resting State Functional Connectivity for Clinicians
}

\author{
Leslie A. Hulvershorn • Kathryn R. Cullen • \\ Michael M. Francis • Melinda K. Westlund
}

Published online: 30 July 2014

(C) Springer International Publishing AG 2014

\begin{abstract}
Resting state functional magnetic imaging (fMRI) is a novel means to examine functional brain networks. It allows investigators to identify functional networks defined by distinct, spontaneous signal fluctuations. Resting state functional connectivity (RSFC) studies examining child and adolescent psychiatric disorders are being published with increasing frequency, despite concerns about the impact of motion on findings. Here we review important RSFC findings on typical brain development and recent publications on child and adolescent psychiatric disorders. We close with a summary of the major findings and current strengths and limitations of RSFC studies.
\end{abstract}

Keywords Functional connectivity $\cdot$ Resting state $\cdot$ Brain development . Functional MRI

\section{Introduction}

For decades, acquiring functional magnetic resonance imaging (fMRI) scans while research participants complete tasks has been the "gold standard" technique for understanding human brain function. However, over the last 5 years, publications and scientific presentations on "resting state fMRI,"

L. A. Hulvershorn $(\bowtie) \cdot$ M. M. Francis

Department of Psychiatry, Indiana University School of Medicine, 705 Riley Hospital Drive, Room 4300, Indianapolis, IN 46205, USA e-mail: lhulvers@iupui.edu

K. R. Cullen

Department of Psychiatry, University of Minnesota, Minneapolis, MN, USA

M. K. Westlund

Department of Psychology, University of Minnesota, Minneapolis, MN, USA "resting state functional connectivity," or "intrinsic functional connectivity" have become quite common. Here we briefly summarize this new brain imaging technique and its relevance to understanding the developing brain. Specifically, we review studies of typical development that have examined how resting state functional connectivity (RSFC) changes across development and in some of the most common psychiatric disorders seen in youth: autism spectrum disorders (ASD), mood and anxiety disorders, attention-deficit/hyperactivity disorder (ADHD), and psychotic disorders. We conclude with a summary and discussion of concerns that have been raised about this novel neuroimaging technique.

\section{What Is Resting State Functional Connectivity?}

RSFC is a term commonly used in the scientific literature to describe a form of fMRI used in research studies where, in contrast to traditional fMRI scan paradigms, there is no specific task for the participants to complete; rather, the scan is acquired while the subject is "at rest." Typically, participants are asked to lie still and to not think about anything in particular. Many studies provide instructions for participants to stay awake with their eyes closed, while others instruct participants to keep their eyes fixed on a neutral stimulus, such as a cross, throughout the scan. The primary principle underlying RSFC is that the pattern of low-frequency fluctuations in the blood oxygen level-dependent (BOLD) signal is highly correlated between brain regions that form functional circuits, even in the absence of an experimental task (Fig. 1) [1••]. Resting state networks discussed in this paper are visually depicted in Fig. 2 [2]. RSFC allows for more facile comparisons across research sites and enables the study of brain function in populations that are typically excluded from task-based MRI studies (i.e., infants and developmentally 


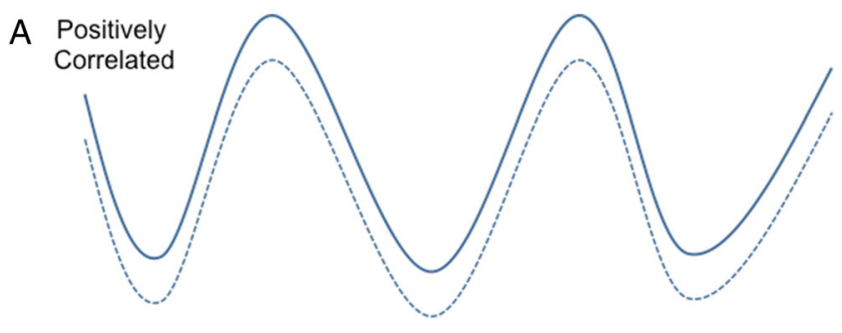

B Anti-correlated

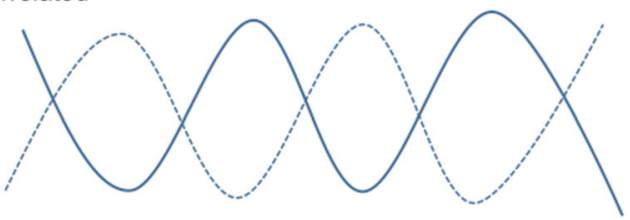

Fig. 1 The waveforms in $\mathbf{A}$ and $\mathbf{B}$ represent the low-frequency blood oxygen level-dependent (BOLD) signal fluctuations (x-axis) originating from two distinct locations in the resting brain, over time (y-axis). A. "Positively" correlated waveforms that are often referred to as "positive resting state functional connectivity." B. Anti-correlated or "negative resting state functional connectivity" between two regions

disabled subjects). Currently, the utility of RSFC is limited to non-clinical research settings.

\section{Typical Development}

\section{Changes During the First 2 Years}

RSFC has been studied in infants as young as 2 weeks of age. The earliest infant study identified five functionally and spatially independent brain networks in 24- to 27-week-old-infants $(n=12)$ [3]. Each of these networks has been described in adults, although the authors noted the absence of one of the best-characterized RSFC networks, the default mode network (DMN). The DMN comprises the medial prefrontal, posterior cingulate, temporal, and parietal cortices, which have been shown to be active, as a network, during rest or during lowcognitive-demand tasks [4]. Theories about the function of the DMN include "the retrieval and manipulation of episodic memories and semantic knowledge" [4], social or self- referential processing [5, 6], or stimulus-independent thought $[7,8]$.

Another study, which compared 2- to 4-week-olds ( $\mathrm{n}=28$ ), 1 -year-olds $(n=26)$, and 2-year-olds $(n=21)$, reported that RSFC strength increased with age, with a more rapid developmental trajectory being noted for sensorimotor as opposed to visual networks [9]. More recently, Gao and colleagues [10] examined RSFC in neonates $(\mathrm{n}=51), 1$-year-olds $(\mathrm{n}=50)$, and 2-year-olds $(\mathrm{n}=46)$, and found that two well-known networks (the DMN and dorsal attention, which is thought to consciously orient attention toward stimuli [11]) evolved from isolated regions in neonates to cohesive networks by the age of 1 year, with further expansion/strengthening of the networks by the age of 2 years [10]. Whereas adult research has shown that these networks are anti-correlated (i.e., the regions oscillate out of sync with each other [12]), this study found that the anti-correlation was absent at birth but became apparent by the age of 1 year and was further enhanced by the age of 2 years [10]. Thus, primitive RSFC networks appear to be present early in infancy but mature rapidly over the first 2 years of life.

Changes from Childhood to Adulthood: Integration, Segregation, Homotopy and Anti-correlations

Several different techniques have been used to study changes in development in RSFC networks from childhood to adulthood. However, findings characterizing RSFC across development must be considered with caution, in light of recent work suggesting that head motion likely impacts and even negates some of these findings, as discussed in more detail below (see "Conclusions"). In a series of studies, Fair and colleagues examined RSFC differences between children, adolescents, and adults. They first examined attention control networks and found that each developmental phase was associated with an increase in long-distance connections (interpreted as improved "integration") and a decrease in short-range connections $[13 \bullet \bullet$. They also found that four networks spanning the brain (DMN, frontoparietal, cinguloopercular, and cerebellar) showed increased integration and segregation across development $[14,15]$. Utilizing a large sample $(n=100)$ of 12 - to 30 -year-olds, Stevens and

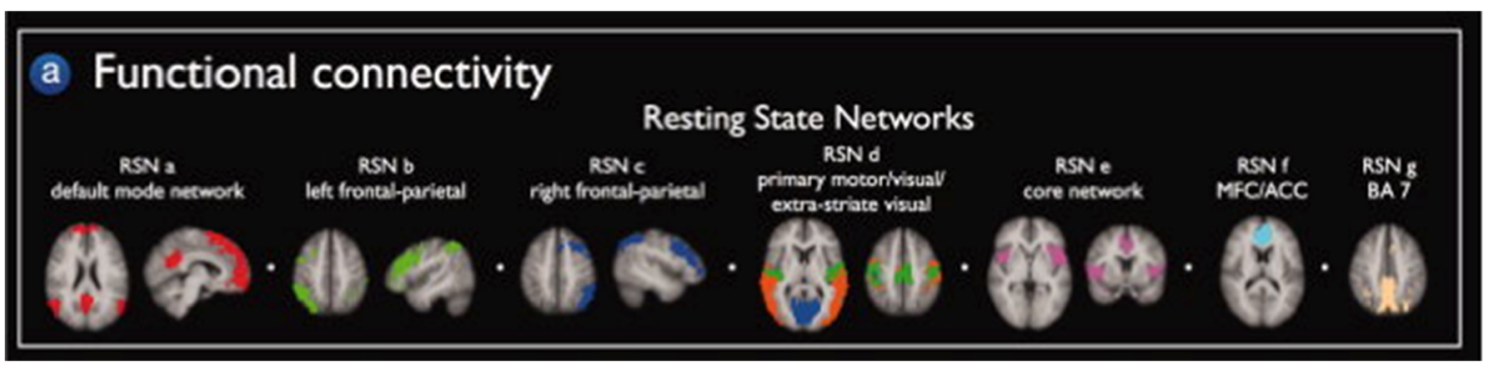

Fig. 2 Group clustering of resting state fMRI data revealed nine resting state networks (RSNs) of functionally linked cortical regions (reproduced with permission from van den Heuvel et al. [2]). ACC anterior cingulate cortex, BA Brodmann area, $M F C$ medial prefrontal cortex 
colleagues also found that increasing age was associated with within-network connectivity growth and more efficient between-network connectivity [16].

Kelly and colleagues examined RSFC development of anterior cingulate cortex (ACC) networks from childhood through adulthood [17]. As in the studies above, they noted a decrease in short-range (local) connectivity with age, and an increase in long-range connectivity. Further, they noted that the greatest degree of developmental change was observed in emotionally relevant regions (originating in the subgenual $\mathrm{ACC}$ [sgACC] and a network of regions involved in social processing (originating in the perigenual ACC [pgACC]). RSFC has also been used to predict individual brain maturity $[18 \bullet, 19]$, with the greatest predictive power being within the cingulo-opercular network, a network though to be involved with cognitive control [20]. Brain maturity was associated with overall weakening of between-network connections and strengthening of within-network connections [18•].

Zuo and colleagues examined homotopy, or the degree to which regions in each hemisphere are connected, of RSFC networks across development [21]. In addition to age-related changes, they found sex differences in homotopy, where females had greater homotopy in the posterior cingulate cortex, medial prefrontal cortex, and middle and superior frontal cortex, but males had greater homotopy in the cerebellum, parahippocampus, and fusiform cortex. Further, there was a sex by age interaction for the dorsolateral prefrontal cortex and amygdala, where females and males showed opposite developmental trajectories.

Narrowing in on specific networks, a recent study examined "task-positive" RSFC networks (the intraparietal sulcus, frontal eye fields, and middle temporal region; regions routinely exhibiting task-related activations [22]; also known as the "ventral attention network") and "task-negative" RSFC networks (similar regions to the DMN; regions routinely exhibiting task-related deactivations [22]) in children $(n=63)$ and adults $(n=28)$ [23]. They found that adults showed greater connectivity between the task-positive network and the dorsolateral prefrontal cortex, and stronger anti-correlations between the task-negative network and the anterior insula, parietal, and posterior cingulate cortices [23]. Chai and colleagues also examined the development of anti-correlated networks in 8- to 24-year-olds [24] and found that as age increased, anticorrelations also increased between networks (i.e., between the medial and dorsolateral prefrontal cortex, and between the lateral parietal cortex and supramarginal gyrus and precuneus). The authors noted that the correlation between regions in these networks was positive in childhood but negative in adulthood, with adolescent connectivity levels falling in the middle [24].

To summarize, across normal development, RSFC networks have been found to span longer distances in the brain and become increasingly segregated, resulting in both within- and between-network efficiency. It appears that particular RSFC networks (i.e., involving the sgACC) are tied to specific developmental functions (i.e., emotional development). Gender differences have received little experimental attention but did appear to be present in studies that examined them [21]. Finally, anti-correlated networks have been reproduced in several studies as markers of more advanced, adult-like development $[23,24]$. All of the studies reviewed here have had the limitation of cross-sectional designs and concerns about the impact of head motion on findings. Longitudinal examinations of developing RSFC networks are needed to definitively characterize changes across development.

\section{Resting State Functional Connectivity Studies with Clinical Samples}

\section{Autism Spectrum Disorders}

Current RSFC research comparing individuals with ASD and healthy comparison participants (with numbers of participants ranging from 20 to 39 for each group) has implicated RSFC abnormalities, including both abnormally high and abnormally low RSFC, in nearly every region of the cortex and the cerebellum [25, 26, 27, 28•].

Studies characterizing specific behavioral problems associated with ASD have largely focused on social and communication deficits. Assaf and colleagues found abnormally decreased RSFC between the precuneus and other DMN areas in ASD patients, compared with controls ( $n=16$ per group) [29]. This atypical connectivity was associated with social and communication deficits [29]. A similar study found that increased RSFC between the posterior cingulate and temporal cortex was associated with social impairment $(n=20$ subjects with ASD; $n=19$ controls) [27]. Furthermore, Abrams and colleagues found that abnormally low RSFC between the temporal cortex and regions of the brain associated with reward was associated with severity of communication deficits among children with ASD ( $n=20$ subjects with ASD; $n=$ 19 controls) $[30 \bullet \bullet$. This finding suggests that communication deficits in ASD may be either the result of, or exacerbated by, reduced ability to associate social interaction with a sense of reward. This is important to consider in the treatment of ASD, as other, more salient, rewarding stimuli may be necessary to advance a patient's social interaction skills. Indeed, this is a practice employed in an applied behavioral analysis (ABA) framework, which emphasizes the use of positive reinforcement in the promotion of social behaviors [31]. Further research examining RSFC before and after initiation of treatments would be of important clinical significance, as RSFC of these regions may normalize over time with interventions. 
Regarding the developmental trajectories of RSFC among children and adolescents with ASD, findings have been inconsistent. One study found that relative to controls $(n=41)$, ASD participants $(n=39)$ had smaller increases in RSFC within the DMN over time [28•], while another found that group differences in DMN RSFC appeared to diminish with age ( $n=40$ subjects with ASD; $n=40$ controls) [32]. Of note, these studies were both cross sectional, and overall functioning and treatment histories varied greatly in the participants in these studies. Future research should carefully consider the influence of these factors and implement longitudinal designs to better understand the developmental trajectory of RSFC in ASD.

\section{Anxiety Disorders}

Studies in this area, while few, have typically taken the approach of examining the association of pediatric anxiety severity with RSFC in healthy participants. Among typically developing children $(n=76)$, Qin and colleagues found that anxiety was associated with increased RSFC between the amygdala and regions involved in emotion perception and regulation and attention [33]. Furthermore, these findings were specific to anxiety symptoms, as other symptoms, such as depressive symptoms, did not show a relationship to RSFC. Also among healthy youth $(n=67)$, anxiety severity was related to increased DMN-insula RSFC [34]. A cross-sectional study found that life stress in infancy was associated with higher childhood cortisol levels and, later, with decreased amygdala-prefrontal RSFC. In turn, amygdala-prefrontal RSFC was inversely related to adolescent anxiety symptoms $(n=57)$ [35]. Thus, anxiety symptoms in healthy samples implicate networks involving the insula, prefrontal cortex, and amygdala.

Roy and colleagues scanned adolescents with generalized anxiety disorder (GAD; $\mathrm{n}=15$ ) and found abnormal amygdala RSFC between the medial prefrontal cortex, insula, and cerebellum, compared with findings in controls $(n=20)[36 \bullet]$. Furthermore, anxiety severity was associated with amygdala RSFC with the insula and superior temporal gyrus. Clearly, further research is needed to replicate these GAD findings and examine other unstudied pediatric anxiety disorders, such as separation anxiety disorder, social anxiety disorder, and specific phobias.

\section{Mood Disorders}

A larger body of literature has used resting state fMRI to examine the neural circuitry underlying mood disorders in children and adolescents. These studies have broadly implicated abnormalities within circuitry that has been shown to be relevant for processing emotions [37]. Specifically, this circuitry encompasses limbic regions such as the amygdala and hippocampus, which are important for both immediate emotional experiences and emotional memory, and regulatory cortical regions such as the prefrontal cortex and the pgACC. Additional regions in this network include the sgACC, which has been strongly implicated in the pathophysiology of mood disorders through the use of several different types of imaging modalities and also postmortem studies [38-40], and the insula, which is known to be important for processing the emotional salience of an individual's experiences. Thus, abnormalities in this circuit could broadly impact emotional processing, resulting in mood states seen in both unipolar and bipolar depressive disorders.

\section{Major Depressive Disorder}

Several studies examining depressed youth have shown abnormalities in sgACC networks, although the pattern of findings has varied. In a small study of mostly medicated adolescents with major depressive disorder [MDD] $(n=12)$ versus controls $(n=14)$, Cullen and colleagues reported that depressed teens showed lower RSFC between the sgACC and a network comprising the dorsal ACC, several frontal and temporal regions, and the insula [41•]. These findings were corroborated in a sample of 36 children (aged 7-11 years), 17 of whom had a history of MDD [42]. However, other studies found greater RSFC between the SgACC and frontal regions in unmedicated adolescents with MDD $(n=23)$ compared with controls $(n=36)$ [43], and in adolescents/young adults with MDD $(n=18)$ compared with controls $(n=20)$ [40]. Furthermore, while Gaffrey and colleagues found greater RSFC between the sgACC and precuneus among children with histories of MDD [42], Connolly et al. found lower RSFC between the sgACC and precuneus in an adolescent MDD group [43]. The divergence of findings in the pattern of sgACC RSFC abnormalities across studies may stem from differences in methodological steps, sample characteristics (e.g., age, pubertal status, age at MDD onset, medication status, comorbidity), or general difficulty in replicability of RSFC findings. Larger studies are needed to clarify the RSFC of the sgACC in pediatric MDD.

Brain regions comprising the striatum (the caudate, putamen, and nucleus accumbens) are thought to play an important role in reward processing, which is known to be impaired in MDD [45]. Gabbay and colleagues examined RSFC centered around the striatum in medication-free adolescents with $\operatorname{MDD}(n=21)$ and healthy controls $(n=21)\left[46^{\circ}\right]$. They reported that the MDD group showed greater RSFC between the striatal regions and the dorsomedial prefrontal cortex and ACC. They also reported a diverse set of connections that were associated with anhedonia [46•]. In contrast, Davey and colleagues found decreased ACC-caudate RSFC in older adolescents with MDD [44]. Although the directionality varied, both studies implicated abnormal frontostriatal neural 
circuits in the pathophysiology of depression, especially with respect to anhedonic symptomatology.

The amygdala is another important brain region investigated in mood-disordered youth. This region is involved in the processing of negative emotion [47] and has consistently been implicated in mood disorders [48]. Luking and colleagues studied amygdala RSFC in a sample of children (aged 7-11 years) with either a history of preschool-onset $\operatorname{MDD}(n=13)$, a maternal history of MDD $(n=11)$, both $(n=13)$, or neither $(n=$ 14) [49]. They found that in comparison with low-risk children, the at-risk groups showed decreased negative amygdala connectivity in a network comprising cortical regulatory regions, but greater positive amygdala connectivity with a network of limbic regions. More recently, Cullen and colleagues examined amygdala RSFC in a large sample of unmedicated adolescents with MDD ( $n=41)$ versus healthy controls [50]. They found that adolescents with MDD showed lower amygdala RSFC with the hippocampus, parahippocampus, and brainstem, but increased amygdala-precuneus RSFC than controls. These findings partially overlapped with the Luking at-risk findings [49], suggesting that amygdala connectivity abnormalities may be present both in childhood and adolescence, before and after the onset of the disorder.

\section{Bipolar Disorder}

Three studies have examined RSFC in youth with bipolar disorder (BD). They used quite different approaches, and each revealed a different aspect of the aberrant RSFC. Dickstein and colleagues examined RSFC stemming from the dorsolateral prefrontal cortex, amygdala, and nucleus accumbens, and found a group difference with dorsolateral prefrontal cortex analysis, in which the BD group $(n=15)$ showed greater negative RSFC with the right superior temporal gyrus, while controls $(n=15)$ had positive RSFC in this circuit [51]. The authors noted that the abnormal frontotemporal circuit is also implicated in memory and learning, and could represent an underlying mechanism for the cognitive deficits involved in BD.

In a more recent paper, $\mathrm{Wu}$ and colleagues used an automated method to examine RSFC across the entire brain [52]. They found that in comparison with controls $(n=40)$, youth with BD $(n=34)$ had greater levels of involvement of the dorsal ACC within affective and executive networks, and greater widespread connectivity within a sensorimotor network. The authors speculated that the excessive involvement of the ACC in both affective and executive networks could explain the association of BD and poor academic performance.

Third, Xiao and colleagues recently studied regional homogeneity (ReHo) in 15 adolescents with BD and 15 healthy controls [53]. Relative to the controls, the subjects with $\mathrm{BD}$ showed lower ReHo in several cortical areas but greater ReHo in several limbic areas. Furthermore, elevated limbic ReHo was correlated with manic symptoms. Thus, three papers, each using distinct methods, have reported cortical-limbic RSFC abnormalities. Caution must be taken in reading the $\mathrm{BD}$ literature, however, given the concern about likely differences between depressed, manic, and euthymic mood states [54]. Considering the difficulty in diagnosing these disorders in youth, additional work with larger, carefully characterized samples is needed.

\section{Combined Mood and Anxiety Disorders}

Depressive and anxiety disorders are often studied together because of their high comorbidity and their categorization as internalizing disorders [55]. Among children with a history of depression and anxiety $(n=30)$, Sylvester and colleagues found reduced RSFC of the task-positive network, compared with findings in healthy controls $(n=42)$ [56]. Another study found that maltreatment in childhood was associated with decreased RSFC between the hippocampus and the sgACC in both males and females with internalizing symptoms at the age of 18 years $(n=64)$ [57••]. Decreased RSFC between the amygdala and the sgACC was also found but only in females $[57 \bullet \bullet$. RSFC in this study was found to mediate the relationship between childhood maltreatment and later internalizing symptoms, suggesting that childhood maltreatment may lead to disrupted connectivity of the fear circuit, which may lead to increased levels of internalizing symptoms.

\section{Attention-Deficit/Hyperactivity Disorder}

Of all psychiatric disorders diagnosed in youth, ADHD has been the best studied, in terms of RSFC. A multi-site datasharing effort, termed ADHD-200 [58•], has recently resulted in publications with very large samples. For example, ADHD brains $(n=757)$ exhibited altered RSFC between the default network and ventral attention networks [59••]. Specifically, diminished anti-correlation was observed between the posterior cingulate cortex (DMN) and the anterior insula and supplementary motor area (ventral attention network). Additionally, the DMN was hypoconnected within-network and had abnormal interconnections between several other networks. This paper replicated similar DMN-related findings from other papers with smaller sample sizes [60, 61]. Given that these regions are involved in directing and sustaining attention, it is logical that they are found to be abnormal in a disorder with inattention as one of its hallmark symptoms.

Clinically meaningful applications of RSFC in ADHD have also begun to appear. Methylphenidate, a primary treatment for ADHD, has been found to influence RSFC of multiple regions, including the dorsolateral prefrontal, parietal, and visual cortices [62] and the DMN and task-positive network [63] in pediatric samples. Similarities between RSFC 
networks have been identified between disorders with shared symptoms (e.g., autism and ADHD) [64], providing neurobiological substantiation for disorder-crossing traits. Clinically observed symptoms have been linked to specific RSFC networks. For example, youth with ADHD and high parent ratings of emotional lability have been found to have abnormal amygdala RSFC, even after accounting for hyperactivity [65]. Finally, RSFC has been used to test models to diagnose ADHD, using brain activity alone, although with mixed success $[66,67]$. Research geared toward clinical applications of RSFC is still in its infancy, but it appears that such studies are likely to emerge in other psychiatric disorders affecting youth, as more publications emerge.

\section{Psychotic Disorders}

The use of fMRI in individuals with psychotic disorders is limited by the cognitive dysfunction associated with these illnesses, making performance on in-scanner experimental tasks difficult to interpret $[68,69]$. Resting state fMRI circumvents the issue of in-scanner task performance and has proven to be a useful tool in schizophrenia research, revealing new information about the functional organization and connectivity of the brain [70-72].

There is increasing interest in studying individuals at ultrahigh risk (UHR) for psychosis or those in their first episode of psychosis (FEP), as they consist of younger individuals with fewer confounding factors associated with chronically ill psychotic populations, such as antipsychotic drug exposure or medical comorbidities. Many studies in these early populations have examined RSFC of the DMN, finding abnormal coherence within DMN structures such as the medial prefrontal cortex, lateral temporal cortex, precuneus, posterior cingulate cortex, and parietal cortex [73-77]. Studies have also examined RSFC between DMN structures and other brain regions, finding abnormal connectivity with task-positive networks [74], as well as the dorsolateral prefrontal cortices [78]. Some of these studies have also examined inter-hemispheric connectivity, finding decreased RSFC in the frontal and temporal regions, and associations between aberrant connectivity and positive and negative symptoms, as well as cognitive dysfunction $[73,79]$.

A study by Lui et al. utilized resting state fMRI to investigate the effects of antipsychotic medication on regional and neural network function in treatment-naïve FEP subjects ( $\mathrm{n}=$ 34), examining RSFC both before and 6 weeks after the initiation of second-generation antipsychotic medication [80•]. The findings revealed that after this short duration of antipsychotic treatment, participants exhibited increased spontaneous regional neural activity in association with symptom improvement, as well as an attenuation of connectivity across widely distributed neural networks. These results may help explain the beneficial effects of antipsychotic medication, hypothetically because of improvements in neurons' ability to function more synchronously with other regions. Importantly, this study highlights the promise that resting state fMRI may hold for strategic development of novel therapeutic agents, as well as for study of biomarkers of patient response to medication in psychotic illness [80•].

\section{Conclusions}

RSFC has been used to study the major child and adolescent psychiatric disorders, albeit with mixed conclusions. The field is still in its infancy, and all findings reviewed here require replication. However, as the methodology continues to advance, RSFC is a promising tool for populations that struggle to comply with tasks and is well suited to large-scale, multisite, and longitudinal studies, such as those characterizing child and adolescent development. Despite these advantages, aspects of RSFC have been met with skepticism among some researchers $[69,81]$, as discussed in more detail below.

\section{Limitation 1: What Are People Actually Doing at} Rest? Interpretation of RSFC findings is subject to the limitation that investigators cannot be sure what their subjects are thinking about or feeling while they are resting. Of particular concern for studies with psychiatric populations, anxiety may be induced by the scanner experience and may be difficult to account for without a "control" task, as in task-based fMRI. Concerns have also been raised that research participants may sleep through scans and may not be aware of when they drift off, even if investigators inquire about this.

Limitation 2: Head Motion Two influential papers documented the serious impact of head motion during the MRI scan on RSFC results [82, 83]. Then, Satterthwaite and colleagues called the validity and replicability of previous developmental connectivity studies into question [84••]. They noted that in prior work on age-related increases in long-distance, within network connectivity and decreases in short-distance, between-network connectivity, the opposite pattern was seen for head motion. They reported that increased head motion is associated with younger age and that when connectivity analyses are conducted with rigorous correction of head motion effects, the developmental findings are still present but substantially tempered. That publication highlighted the importance of correcting for head motion in developmental studies of RSFC.

In summary, clinicians will likely encounter publications or presentations utilizing RSFC, an innovative fMRI technique. RSFC refers to spontaneous brain fluctuations, organized into functional communication networks, which are quantifiable when subjects are at rest. RSFC can reveal novel information 
about how brain networks develop and how that development can go awry in various psychiatric disorders. The RSFC literature is still at an early stage, as illustrated by the fact that vast networks of abnormalities have been implicated for most disorders. Few findings have been replicated; this may be because many of the initial studies have included small samples and the methodologies to process and analyze the data have varied widely. RSFC is a technique that is quite susceptible to motion artifact, leading to some recent skepticism about the initial findings from pediatric studies, given the large degree of motion in children and adolescent participants. However, recent work has incorporated more robust techniques to address motion. Moving forward, studies with larger samples that incorporate uniform methods across studies will be most useful for advancing the field. There is strong interest in gaining insight into how RSFC changes across normal and abnormal development, thus longitudinal research is needed. RSFC is likely to continue to serve as an important neuroimaging modality as findings are reproduced, clinical interventions are tested, and analytic techniques improve.

\section{Compliance with Ethics Guidelines}

Conflict of Interest Kathryn Cullen and Leslie Hulvershorn received grants from the National Institutes of Health, the Brain and Behavior Research Foundation (formerly NARSAD). Kathryn Cullen also received funding from the Center for Translational Science Institute at the University of Minnesota, and the Academic Center at the University of Minnesota; and travel expenses covered by a National Institutes of Mental Health grant. Michael Francis received research support from the Brain and Behavior Research Foundation and Neuronetics. Melinda Westlund has no conflicts of interest.

Human and Animal Rights and Informed Consent This article does not contain any studies with human or animal subjects performed by the authors.

\section{References}

Papers of particular interest, published recently, have been highlighted as:

- Of importance

•- Of major importance

1.• Biswal B et al. Functional connectivity in the motor cortex of resting human brain using echo-planar MRI. Magn Reson Med: Off J Soc Magn Reson Med Soc Magn Reson Med. 1995;34(4): 537-41. Landmark paper which first described the concept of RSFC.

2. van den Heuvel MP, Mandl RCW, Kahn RS, Hulshoff Pol HE. Functionally linked resting-state networks reflect the underlying structural connectivity architecture of the human brain. Hum Brain Mapp. 2009;30(10):3127-41.
3. Fransson $\mathrm{P}$ et al. Resting-state networks in the infant brain. Proc Natl Acad Sci U S A. 2007;104(39):15531-6.

4. Greicius MD et al. Functional connectivity in the resting brain: a network analysis of the default mode hypothesis. Proc Natl Acad Sci U S A. 2003;100(1):253-8.

5. Uddin LQ, Rayman J, Zaidel E. Split-brain reveals separate but equal self-recognition in the two cerebral hemispheres. Conscious Cogn. 2005;14(3):633-40.

6. Buckner RL, Carroll DC. Self-projection and the brain. Trends Cogn Sci. 2007;11(2):49-57.

7. Mason MF et al. Wandering minds: the default network and stimulus independent thought. Science. 2007;315(5810):393-5.

8. McKiernan KA et al. Interrupting the "stream of consciousness": an fMRI investigation. NeuroImage. 2006;29(4):1185-91.

9. Lin $\mathrm{W}$ et al. Functional connectivity MR imaging reveals cortical functional connectivity in the developing brain. AJNR Am J Neuroradiol. 2008;29(10):1883-9.

10. Gao $\mathrm{W}$ et al. The synchronization within and interaction between the default and dorsal attention networks in early infancy. Cereb Cortex. 2013;23(3):594-603.

11. Ozaki TJ. Frontal-to-parietal top-down causal streams along the dorsal attention network exclusively mediate voluntary orienting of attention. PLoS One. 2011;6(5):e20079.

12. Raichle ME et al. A default mode of brain function. Proc Natl Acad Sci U S A. 2001;98(2):676-82.

13.• Fair DA et al. Development of distinct control networks through segregation and integration. Proc Natl Acad Sci U S A. 2007;104(33):13507-12. This study examined RSFC in children, adolescents and adults and found that each developmental phase was associated with an increase in long-distance connections and an increase in short-distance connections.

14. Fair DA et al. The maturing architecture of the brain's default network. Proc Natl Acad Sci U S A. 2008;105(10):4028-32.

15. Fair DA et al. Functional brain networks develop from a "local to distributed" organization. PLoS Comput Biol. 2009;5(5): e1000381.

16. Stevens MC, Pearlson GD, Calhoun VD. Changes in the interaction of resting-state neural networks from adolescence to adulthood. Hum Brain Mapp. 2009;30(8):2356-66.

17. Kelly AM et al. Development of anterior cingulate functional connectivity from late childhood to early adulthood. Cereb Cortex. 2009;19(3):640-57.

18. Dosenbach NU et al. Prediction of individual brain maturity using fMRI. Science. 2010;329(5997):1358-61. This study showed that RSFC can reliably be used to predict "brain age."

19. Wang $\mathrm{L}$ et al. Decoding lifespan changes of the human brain using restingstate functional connectivity MRI. PLoS One. 2012;7(8): e44530.

20. Sestieri $\mathrm{C}$ et al. Domain-general signals in the cingulo-opercular network for visuospatial attention and episodic memory. J Cogn Neurosci. 2014;26(3):551-68.

21. Zuo XN et al. Growing together and growing apart: regional and sex differences in the lifespan developmental trajectories of functional homotopy. J Neurosci. 2010;30(45):15034-43.

22. Fox MD et al. The human brain is intrinsically organized into dynamic, anticorrelated functional networks. Proc Natl Acad Sci U S A. 2005;102(27):9673-8.

23. Barber AD et al. Developmental changes in within- and betweennetwork connectivity between late childhood and adulthood. Neuropsychologia. 2013;51(1):156-67.

24. Chai XJ et al. Selective development of anticorrelated networks in the intrinsic functional organization of the human brain. J Cogn Neurosci. 2014;26(3):501-13.

25. Maximo JO et al. Approaches to local connectivity in autism using resting state functional connectivity MRI. Front Hum Neurosci. 2013;7:605. 
26. Paakki JJ et al. Alterations in regional homogeneity of resting-state brain activity in autism spectrum disorders. Brain Res. 2010;1321: 169-79.

27. Lynch CJ et al. Default mode network in childhood autism: posteromedial cortex heterogeneity and relationship with social deficits. Biol Psychiatry. 2013;74(3):212-9.

28. Wiggins JL et al. Using a self-organizing map algorithm to detect age-related changes in functional connectivity during rest in autism spectrum disorders. Brain Res. 2011;1380:187-97. Study of the difference in developmental changes of RSFC between youth with ASD and healthy controls.

29. Assaf $\mathrm{M}$ et al. Abnormal functional connectivity of default mode sub-networks in autism spectrum disorder patients. Neuroimage. 2010;53(1):247-56

30.• Abrams DA et al. Underconnectivity between voice-selective cortex and reward circuitry in children with autism. Proc Natl Acad Sci U S A. 2013;110(29):12060-5. RSFC and ASD study that integrates anomalous RSFC involving reward circuitry with communication deficits, which corresponds well with current treatment approaches for ASD.

31. Lovaas OI. Behavioral treatment and normal educational and intellectual functioning in young autistic children. J Consult Clin Psychol. 1987;55(1):3-9.

32. Anderson JS et al. Functional connectivity magnetic resonance imaging classification of autism. Brain. 2011;134(Pt 12):3742-54.

33. Qin S, et al. Amygdala subregional structure and intrinsic functional connectivity predicts individual differences in anxiety during early childhood. Biol Psychiatry. 2013.

34. Dennis EL et al. Anxiety modulates insula recruitment in restingstate functional magnetic resonance imaging in youth and adults. Brain Connect. 2011;1(3):245-54.

35. Burghy CA et al. Developmental pathways to amygdala-prefrontal function and internalizing symptoms in adolescence. Nat Neurosci. 2012;15(12):1736-41

36. Roy AK et al. Intrinsic functional connectivity of amygdala-based networks in adolescent generalized anxiety disorder. J Am Acad Child Adolesc Psychiatry. 2013;52(3):290-299 e2. First study examining amygdala RSFC among youth with GAD compared to controls and associating amygdala RSFC with anxiety severity.

37. Hulvershorn LA, Cullen K, Anand A. Toward dysfunctional connectivity: a review of neuroimaging findings in pediatric major depressive disorder. Brain Imaging Behav. 2011;5(4):307-28.

38. Drevets WC, Ongur D, Price JL. Reduced glucose metabolism in the subgenual prefrontal cortex in unipolar depression. Mol Psychiatry. 1998;3(3):190-1.

39. Drevets WC, Savitz J, Trimble M. The subgenual anterior cingulate cortex in mood disorders. CNS Spectr. 2008;13(8):663-81.

40. Mayberg HS et al. Deep brain stimulation for treatment-resistant depression. Neuron. 2005;45(5):651-60.

41. Cullen KR et al. A preliminary study of functional connectivity in comorbid adolescent depression. Neurosci Lett. 2009;460(3):22731. In the first publication of RSFC in adolescent depression, this study showed that depressed adolescents had abnormally low $R S F C$ in a network based in th sgACC, an area previously found to have abnormal functioning and size in depression.

42. Gaffrey MS et al. Subgenual cingulate connectivity in children with a history of preschool-depression. Neuroreport. 2010;21(18):1182-8.

43. Connolly CG et al. Resting-state functional connectivity of subgenual anterior cingulate cortex in depressed adolescents. Biol Psychiatry. 2013;74(12):898-907.

44. Davey CG et al. Regionally specific alterations in functional connectivity of the anterior cingulate cortex in major depressive disorder. Psychol Med. 2012;42(10):2071-81.

45. Phillips ML et al. Neurobiology of emotion perception I: the neural basis of normal emotion perception. Biol Psychiatry. 2003;54(5): 504-14.
46. Gabbay V et al. Striatum-based circuitry of adolescent depression and anhedonia. J Am Acad Child Adolesc Psychiatry. 2013;52(6): 628-41 e13. This study found abnormal frontal-striatal RSFC in adolescents with depression. This abnormality was related to symptoms of anhedonia.

47. Phelps EA, LeDoux JE. Contributions of the amygdala to emotion processing: from animal models to human behavior. Neuron. 2005;48(2):175-87.

48. Drevets WC. Neuroimaging abnormalities in the amygdala in mood disorders. Ann N Y Acad Sci. 2003;985:420-44.

49. Luking KR et al. Functional connectivity of the amygdala in earlychildhoodonset depression. J Am Acad Child Adolesc Psychiatry. 2011;50(10):1027-41 e3.

50. Cullen KR, et al. Abnormal amygdala resting -state functional connectivity in adolescent depression. JAMA Psychiatry. in Press.

51. Dickstein DP et al. Fronto-temporal spontaneous resting state functional connectivity in pediatric bipolar disorder. Biol Psychiatry. 2010;68(9):839-46.

52. $\mathrm{Wu} \mathrm{M}$ et al. Altered affective, executive and sensorimotor resting state networks in patients with pediatric mania. J Psychiatry Neurosci. 2013;38(4):232-40.

53. Xiao Q et al. Altered regional homogeneity in pediatric bipolar disorder during manic state: a resting-state fMRI study. PLoS One. 2013;8(3):e57978.

54. Hulvershorn LA et al. Neural activation during facial emotion processing in unmedicated bipolar depression, euthymia, and mania. Biol Psychiatry. 2012;71(7):603-10.

55. Krueger RF. The structure of common mental disorders. Arch Gen Psychiatry. 1999;56(10):921-6.

56. Sylvester $\mathrm{CM}$ et al. Resting state functional connectivity of the ventral attention network in children with a history of depression or anxiety. J Am Acad Child Adolesc Psychiatry. 2013;52(12): 1326-1336 e5.

$57 . \bullet$ Herringa RJ et al. Childhood maltreatment is associated with altered fear circuitry and increased internalizing symptoms by late adolescence. Proc Natl Acad Sci U S A. 2013;110(47):19119-24. A study examining the effects of childhood maltreatment on later internalizing symptoms (i.e., depression and anxiety) and RSFC in adolescence. This study suggests important environmental effects on developmental psychopathology.

58. The ADHD-200 Consortium. A model to advance the translational potential of neuroimaging in clinical neuroscience. Front Syst Neurosci. 2012;6:62. A description of an innovative RSFC data sharing project.

$59 . \bullet$ Sripada C, et al. Disrupted network architecture of the resting brain in attentiondeficit/ hyperactivity disorder. Hum Brain Mapp. 2014. A product of the ADHD-200 Consortium, a large, multi-site examination of RSFC in ADHD that replicated prior findings of smaller samples.

60. Sun L et al. Abnormal functional connectivity between the anterior cingulate and the default mode network in drug-naive boys with attention deficit hyperactivity disorder. Psychiatry Res. 2012;201(2):120-7.

61. Chabernaud $\mathrm{C}$ et al. Dimensional brain-behavior relationships in children with attention-deficit/hyperactivity disorder. Biol Psychiatry. 2012;71(5):434-42.

62. Yang $\mathrm{H}$ et al. Abnormal spontaneous brain activity in medicationnaive ADHD children: a resting state fMRI study. Neurosci Lett. 2011;502(2):89-93.

63. An L et al. Methylphenidate normalizes resting-state brain dysfunction in boys with attention deficit hyperactivity disorder. Neuropsychopharmacol: Off Publ Am Coll Neuropsychopharmacol. 2013;38(7):1287-95.

64. Di Martino A et al. Shared and distinct intrinsic functional network centrality in autism and attention-deficit/hyperactivity disorder. Biol Psychiatry. 2013;74(8):623-32. 
65. Hulvershorn LA et al. Abnormal amygdala functional connectivity associated with emotional lability in children with attention-deficit/ hyperactivity disorder. J Am Acad Child Adolesc Psychiatry. 2014;53(3):351-361 e1.

66. Brown MR et al. ADHD-200 Global Competition: diagnosing ADHD using personal characteristic data can outperform resting state fMRI measurements. Front Syst Neurosci. 2012;6:69.

67. Bohland JW et al. Network, anatomical, and non-imaging measures for the prediction of ADHD diagnosis in individual subjects. Front Syst Neurosci. 2012;6:78.

68. Venkataraman A et al. Whole brain resting state functional connectivity abnormalities in schizophrenia. Schizophr Res. 2012;139(1-3):7-12.

69. Greicius M. Resting-state functional connectivity in neuropsychiatric disorders. Curr Opin Neurol. 2008;21(4):424-30.

70. Biswal B et al. Functional connectivity in the motor cortex of resting human brain using echo-planar MRI. Magn Reson Med. 1995;34(4):537-41.

71. Gusnard DA, Raichle ME, Raichle ME. Searching for a baseline: functional imaging and the resting human brain. Nat Rev Neurosci. 2001;2(10):685-94.

72. Peltier SJ, Polk TA, Noll DC. Detecting low-frequency functional connectivity in fMRI using a self-organizing map (SOM) algorithm. Hum Brain Mapp. 2003;20(4):220-6.

73. Guo W et al. Decreased resting-state interhemispheric coordination in firstepisode, drug-naive paranoid schizophrenia. Prog Neuropsychopharmacol Biol Psychiatry. 2014;48:14-9.

74. Shim G et al. Altered resting-state connectivity in subjects at ultrahigh risk for psychosis: an fMRI study. Behav Brain Funct. 2010;6:58.

75. Alonso-Solis A et al. Altered default network resting state functional connectivity in patients with a first episode of psychosis. Schizophr Res. 2012;139(1-3):13-8.

76. Lui $\mathrm{S}$ et al. Association of cerebral deficits with clinical symptoms in antipsychotic-naive first-episode schizophrenia: an optimized voxel-based morphometry and resting state functional connectivity study. Am J Psychiatry. 2009;166(2):196-205.

77. Guo W et al. Abnormal default-mode network homogeneity in first-episode, drug-naive schizophrenia at rest. Prog NeuroPsychopharmacol Biol Psychiatry. 2014;49:16-20.

78. Zhou Y et al. Functional dysconnectivity of the dorsolateral prefrontal cortex in first-episode schizophrenia using resting-state fMRI. Neurosci Lett. 2007;417(3):297-302.

79. Mwansisya TE et al. The diminished interhemispheric connectivity correlates with negative symptoms and cognitive impairment in first-episode schizophrenia. Schizophr Res. 2013;150(1):144-50.

80. Lui S et al. Short-term effects of antipsychotic treatment on cerebral function in drug-naive first-episode schizophrenia revealed by "resting state" functional magnetic resonance imaging. Arch Gen Psychiatry. 2010;67(8):783-92. An interesting study of the effect of second-generation antipsychotic medication on resting state functional connectivity in medication-naïve individuals with firstepisode psychosis.

81. Leibenluft E, Pine DS. Resting state functional connectivity and depression: in search of a bottom line. Biol Psychiatry. 2013;74(12):868-9.

82. Power JD et al. Spurious but systematic correlations in functional connectivity MRI networks arise from subject motion. Neuroimage. 2012;59(3):2142-54.

83. Van Dijk KR, Sabuncu MR, Buckner RL. The influence of head motion on intrinsic functional connectivity MRI. Neuroimage. 2012;59(1):431-8.

84.• Satterthwaite TD et al. Impact of in-scanner head motion on multiple measures of functional connectivity: relevance for studies of neurodevelopment in youth. Neuroimage. 2012;60(1):623-32. An influential paper, which called into question the concern about head motion, particularly in developmental RSFC studies. 